\title{
Universiteit
}

Leiden

The Netherlands

\section{A revised bacterial polypeptide chain elongation cycle with a stepwise increase in restriction of unwanted ternary complexes by the ribosome}

Bosch, L.; Vijgenboom, E.; Zeef, L.A.H.

\section{Citation}

Bosch, L., Vijgenboom, E., \& Zeef, L. A. H. (1996). A revised bacterial polypeptide chain elongation cycle with a stepwise increase in restriction of unwanted ternary complexes by the ribosome. Biochemistry, 35(39), 12647-12651. doi:10.1021/bi952925a

Version: $\quad$ Publisher's Version

License: $\quad$ Licensed under Article 25fa Copyright Act/Law (Amendment Taverne)

Downloaded from: https://hdl.handle.net/1887/3238805

Note: To cite this publication please use the final published version (if applicable). 


\title{
A Revised Bacterial Polypeptide Chain Elongation Cycle with a Stepwise Increase in Restriction of Unwanted Ternary Complexes by the Ribosome ${ }^{\dagger}$
}

\author{
Leendert Bosch,* Erik Vijgenboom, and Leo A. H. Zeef* \\ Department of Biochemistry, Leiden Institute of Chemistry, Gorlaeus Laboratories, Leiden University, The Netherlands
}

Received December 12, 1995; Revised Manuscript Received July 2, 1996

The polypeptide chain elongation cycle and the decoding of the genetic message in Escherichia coli have been a matter of general consensus during the last twenty-five years (Miller \& Weissbach, 1977; Kaziro, 1990). Some years ago, however, when we were studying the in vivo effects of

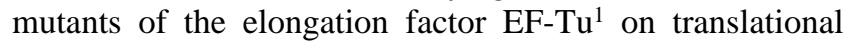
errors (Vijgenboom et al., 1985; Vijgenboom \& Bosch, 1989), serious doubts arose in our minds concerning the correctness of the classical elongation scheme. We found that some mutant EF-Tu species act as suppressors of nonsense codons and translational frame shifts. Surprisingly, optimal suppression required the combined action of two different mutant EF-Tu species. On account of these and later findings, we now propose a revised elongation cycle in which two molecules of EF-Tu operate successively and in which the restriction by the ribosome of unwanted ternary complexes increases stepwise.

\section{SUPPRESSION OF NONSENSE AND FRAME SHIFTS BY MUTANTS OF EF-TU}

Intergenic suppression of nonsense mutations can be mediated by suppressor genes coding for a tRNA or a

$\dagger$ L.B. is supported by the EC in the framework of the Human Capital and Mobility Programme.

* Author to whom correspondence should be addressed. L. Bosch, Department of Biochemistry, Leiden Institute of Chemistry, Gorlaeus Laboratories, P.O. Box 9502, 2300 RA Leiden, The Netherlands. Telephone: 31-71-5274763. Fax: 31-71-5274537.

$\$$ Present address: Section of Biochemistry, Brown University, Providence, RI.

${ }^{1}$ Abbreviations: aa-tRNA, aminoacyl-tRNA; EF-G, elongation factor G; EF-Ts, elongation factor Ts; EF-Tu, elongation factor Tu; EF-TuA $A_{R}$, [A375T]EF-TuA; EF-TuB, , [G222D]EF-TuB; tc, ternary complex of EF-Tu $\cdot G T P \cdot a a-t R N A$. ribosomal protein (Ozeki et al., 1980; Gorini, 1974). Translational stops at the codons UAG and UAA can also be suppressed in cells harboring mutant species of EF-Tu as was demonstrated by Vijgenboom et al. (1985). Readthrough of these codons at position 189 of the lacI- $Z$ fusion on an $\mathrm{F}^{\prime}$ factor was determined by measuring $\beta$-galactosidase activity in these mutant cells. The EF-Tu species active in suppression are [A375T]EF-TuA and [G222D]EF-TuB. For historical reasons, they are designated here EF-Tu $A_{R}$ and $\mathrm{EF} \mathrm{TuB} \mathrm{B}_{\mathrm{o}}$, respectively. These mutant factors also promote +1 and -1 translational frame shifts. Vijgenboom and Bosch (1989) determined this phenomenon in E. coli strains transformed with plasmids carrying a frame shift mutation in a cII-lacZ fusion.

$\mathrm{EF}-\mathrm{TuA}_{\mathrm{R}}$ has a low affinity for kirromycin (van de Klundert et al., 1978). It is error-prone (van der Meide et al., 1980; Vijgenboom et al., 1985; Vijgenboom \& Bosch, 1989) and somewhat less effective than wild-type EF-Tu in nucleotide binding (Duisterwinkel et al., 1981) and in ternary complex formation (Swart et al., 1987). Its activity to mediate in vitro translation of a natural mRNA is reduced (Talens et al., to be published). When present as the sole EF-Tu species in the cell, EF-TuA $A_{R}$ does not display readthrough of nonsense codons above the wild-type level.

EF-TuB $B_{\mathrm{o}}$ is encoded by the gene $t u f B_{\mathrm{o}}$, which is recessive to $t u f A_{R}$ encoding $\mathrm{EF}_{-\mathrm{Tu}} \mathrm{A}_{\mathrm{R}}$ and conferring kirromycin resistance (van de Klundert et al., 1978; Tapio \& Kurland, 1986). Studies by Duisterwinkel et al. (1981) and by Swart et al. (1987) showed that EF-TuB is able to form a ternary complex and can transfer Phe-tRNA to poly(U)-programmed ribosomes. The interaction with the ribosome is defective; however, both triggering of the GTPase and poly(Phe) synthesis require elevated $\mathrm{Mg}^{2+}$ concentrations $(10-14 \mathrm{mM})$. 
At the $\mathrm{Mg}^{2+}$ concentrations optimal for wild-type EF-Tudependent poly(Phe) synthesis, EF-TuB ${ }_{o}$ is inactive. Recent experiments by Talens et al. (to be published) demonstrate that $\mathrm{EF}^{-} \mathrm{TuB}_{\mathrm{o}}$ virtually lacks any activity to sustain in vitro translation of a natural messenger RNA at $\mathrm{Mg}^{2+}$ concentrations up to $15 \mathrm{mM}$. Evidence for a defective interaction with the ribosome was presented by Swart et al. (1987), who reported that the ribosome-dependent stimulation of the EF$\mathrm{TuB}_{\mathrm{o}}$ GTPase requires codon-anticodon interaction in contrast to the wild-type EF-Tu GTPase which does not display such a requirement. Surprisingly, EF-TuB drasti- $^{-}$ cally enhances suppression of nonsense codons and of translational frame shifts when present in cells together with $\mathrm{EF}-\mathrm{Tu}_{R}$. Since EF-TuB $\mathrm{B}_{\mathrm{o}}$ by itself does not support protein synthesis, this leads to the conclusion that the intracellular presence of both EF-TuAR and $\mathrm{EF}-\mathrm{TuB}_{\mathrm{O}}$ is a prerequisite of nonsense suppression [see also Hughes (1987) and Hughes et al. (1987)]. No suppression was observed in cells containing wild-type EF-Tu and either one of the two mutant factors.

$\mathrm{EF} \mathrm{Tu} \mathrm{A}_{\mathrm{R}}$ has a limited ability to induce frame shifts, as becomes apparent in cells harboring this factor as the sole EF-Tu. A linear relationship exists between the frequency of frame shifting and the cellular concentration of (chromosome- or plasmid-borne) EF-TuA $A_{R}$ (Vijgenboom \& Bosch, 1989). EF-TuA $A_{R}$ molecules alone thus do not show cooperativity. Cells containing both $\mathrm{EF}^{-T u A_{R}}$ and $\mathrm{EF}^{-T u B_{o}}$, in amounts comparable to the wild-type level, display maximal frame shifting. Here again, the two mutant factors display a remarkable synergism.

The striking feature of these data is that $\mathrm{EF}_{-} \mathrm{TuB}_{0}$, unable to mediate translation of natural mRNA, contributes significantly to translation (albeit mistranslation) when EF-TuA $A_{R}$ is also present. If these data obtained with mutant EF-Tu have relevance for elongation mediated by wild-type factor, the possibility may be envisaged that also in the wild-type cell two molecules of EF-Tu operate during each round of the elongation cycle. In 1990, Ehrenberg et al. suggested on the basis of kinetic results that aa-tRNA is recruited as a quinary complex containing two molecules of EF-Tu and two of GTP [see also Ehrenberg et al. (1993) and Scoble et al. (1994)]. From their studies, the authors concluded further that two molecules of GTP are hydrolyzed for each aa-tRNA delivered to the ribosome. Weijland and Parmeggiani (1993, 1994), using an engineered EF-Tu with modified substrate specificity, elegantly demonstrated that two GTP molecules are hydrolyzed, indeed, for each peptide bond formed. Raising the question of whether this hydrolysis occurs synchronously or sequentially, they pointed out (1994) that synchronous hydrolysis requires triggering of EF-Tu GTPases by two distinct ribosomal sites, which cannot be directly covered by the $\alpha$-sarcin loop. They therefore favor a sequential mechanism (see also below).

The synergistic suppressor activities of EF-TuA $A_{R}$ and EF$\mathrm{TuB}_{\mathrm{o}}$ seem to be in line with the proposed quinary complex and a synchronous hydrolysis of two GTP molecules. Intermolecular interactions of $\mathrm{EF}^{-T u A_{R}}$ and $\mathrm{EF}^{-T u B_{o}}$ in vitro have been observed by Swart (1987) and by Anborgh and Parmeggiani (1991). Bensch et al. (1991), however, using a variety of methods could not confirm the existence of a quinary complex. Ehrenberg et al. (1993) later restricted the existence of this complex to temperatures higher than $20^{\circ} \mathrm{C}$. Below, we argue that two sequential EF-Tu-mediated
GTP hydrolysis steps may offer an explanation for the high accuracy of protein synthesis. We propose that suppression also occurs in two successive stages. Due to the inability of EF-TuB ${ }_{0}$ to mediate translation of natural mRNA by itself, the first GTP may then be hydrolyzed by EF-TuA $A_{R}$, the second by $\mathrm{EF}^{-T u B_{0}}$. We indicate that such a sequence of events is not in conflict with the lack of suppressor activity exhibited by the combination of wild-type EF-Tu and EF$\mathrm{TuB}_{\mathrm{o}}$ (Vijgenboom et al., 1985; Vijgenboom \& Bosch, 1989). This leads us to propose a revised model for the polypeptide chain elongation cycle in $E$. coli in which EF-Tu molecules operate in two separate steps.

\section{A REVISED MODEL FOR POLYPEPTIDE CHAIN ELONGATION}

The revised scheme for polypeptide chain elongation is outlined in Figure 1. Step 1 takes place posttranslocationally when peptidyl-tRNA is located at the ribosomal P site and a deacylated tRNA is in the ribosomal E site. Weijland and Parmeggiani (1994) have recently considered the possibility that the hydrolysis by EF-Tu of one of the two GTP molecules could lead to the ejection of the deacylated tRNA from the E site (step 2). They refer to the report by Triana et al. (1993) that vacating of the E site in fungi in the posttranslocational state also requires GTP (or ATP). In $E$. coli, 41 different ternary complexes (tc's) compete for an empty site on the ribosome after translocation. Most of these encounters do not result in codon-anticodon interaction because 36 or 37 out of the 41 tc's are noncognate and are unable to interact with the mRNA (interaction of cognate and near-cognate tc's with mRNA is discussed below). Since occupation of the $\mathrm{E}$ site lowers the affinity of the A site for the tc's (Nierhaus, 1993), the activation of the EF-Tu GTPase is low but sufficient to induce GTP hydrolysis even in the absence of codon-anticodon interaction as was demonstrated by Swart et al. (1987) (see also below where the role of the ribosome in inducing GTP hydrolysis in steps 1 and 2 versus steps 3 and 4 is discussed in more detail). GTP hydrolysis results in a conformational change of the ribosome, release of aa-tRNA and of EF-Tu·GDP, and emptying of the ribosomal E site (step 2). According to the allosteric linkage of the $\mathrm{E}$ and $\mathrm{A}$ sites, the conformation of the A site will be altered significantly (Nierhaus, 1993).

The initial steps 1 and 2 of the elongation process, in which any noncognate tc may be involved, cannot be carried out by EF-TuB ${ }_{0} \cdot G T P \cdot a a-t R N A$ since, as pointed out above, it requires codon-anticodon interaction. On the other hand, EF-TuA $A_{R} \cdot G T P \cdot a a-t R N A$ is capable of doing so. This paves the way for $\mathrm{EF}^{-T u B} \cdot \mathrm{B}_{0} \cdot \mathrm{GTP} \cdot \mathrm{aa}-\mathrm{tRNA}$ to perform the second GTP-consuming reaction (Figure 1, steps 3 and 4) which is dependent on codon-anticodon interaction. Here we wish to emphasize the different role of the ribosome in activating the EF-Tu GTPase center in the presence (steps 3 and 4) and absence (steps 1 and 2) of codon-anticodon interaction ("coded" versus "non-coded" activation). Coded activation occurs on ribosomes with an empty E site and non-coded activation on ribosomes with deacylated tRNA in the E site. Previous studies (Ruusala et al., 1982a,b; Rodnina et al., 1995) performed with ribosomes with a vacant $E$ site demonstrated that the activation of the EF-Tu GTPase is strictly dependent on codon-anticodon interaction. Noncoded activation has been observed by Swart et al. (1982), 

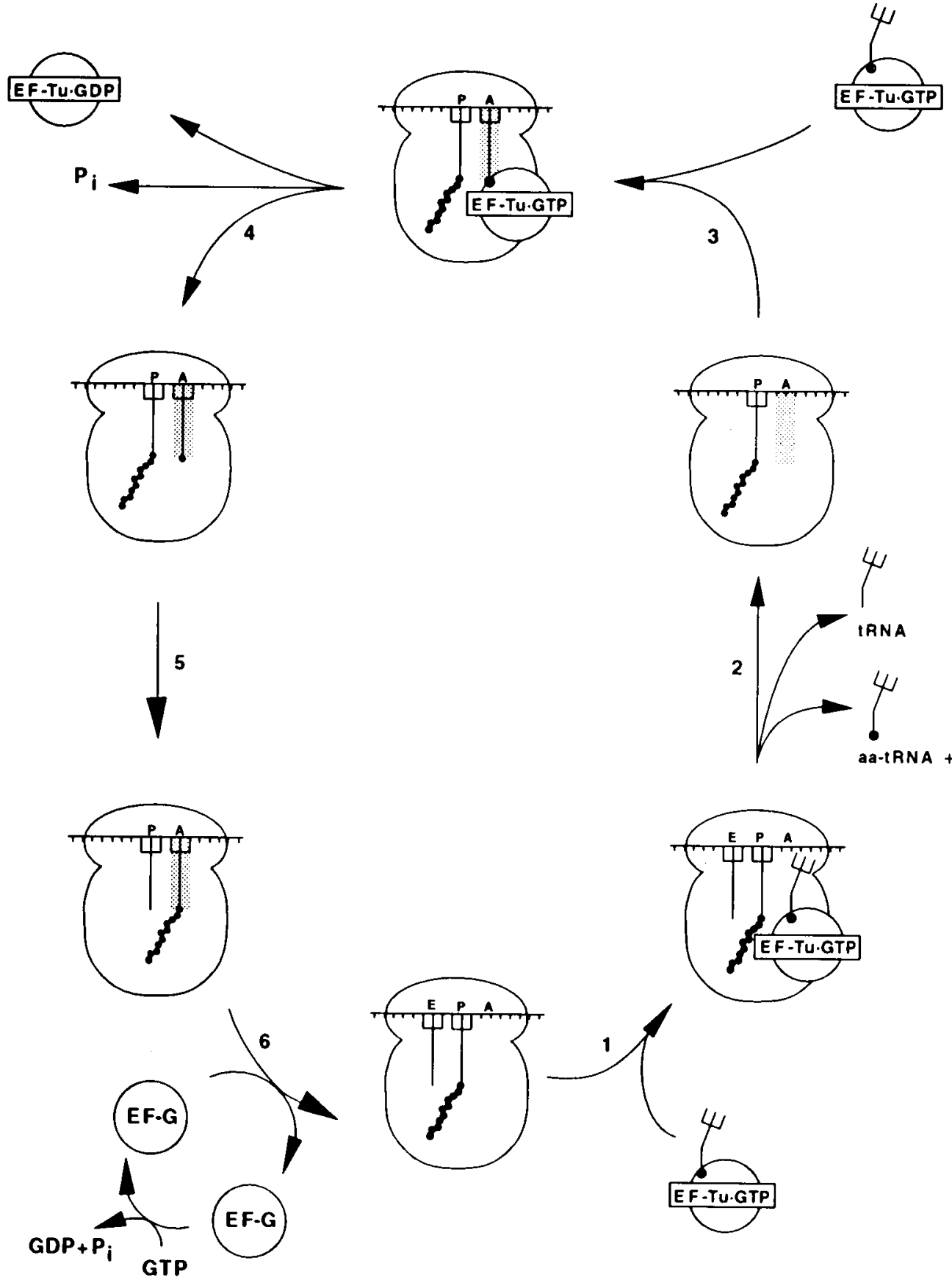

FIGURE 1: Revised polypeptide chain elongation cycle. (Step 1) The ternary complex (tc) enters the ribosome in the post-translocational state. Most of the tc's (90\%) are noncognate and do not interact with the mRNA (see text). (Step 2) Interaction with the ribosome triggers GTP hydrolysis. As a result, aa-tRNA and EF-Tu•GDP are released from the A site and deacylated tRNA is released from the E site. Due to the allosteric linkage of $\mathrm{E}$ and $\mathrm{A}$ sites, the latter site undergoes a conformational change and becomes highly restrictive (shaded A site). (Step 3) A tc carrying a cognate or near-cognate tRNA enters the ribosome $\cdot m R N A$ complex. Codon-anticodon interaction alters the tRNA conformation, which is a condition for binding of the tc to the restrictive A site and the hydrolysis of a second GTP molecule. Noncognate tRNAs are excluded, due to failing codon-anticodon interaction (ribosomal screen). (Step 4) Conformational discrimination between cognate and near-cognate tRNAs and proofreading. Hydrolysis of a second GTP molecule and release of a second EF-Tu GDP complex. (Step 5) Peptide bond formation and (step 6) translocation do not differ from the classical scheme.

in a translating system lacking EF-G (in which all elongation steps, including vacating of the E site, are extremely slow).

tRNA-mRNA interaction alters the conformation of a cognate tRNA (Kurland, 1979) and to a lesser extent that of a near-cognate tRNA. This will affect not only the interaction of a tc with the ribosomal-decoding region but also that with the ribosome outside this region. This conformational alteration enables $\mathrm{EF}-\mathrm{TuB}_{\mathrm{o}} \cdot \mathrm{GTP} \cdot \mathrm{aa}-\mathrm{tRNA}$ to interact productively with the ribosomal A site. Apparently, it fulfills the requirements for interaction with the A site which at this stage of the cycle have become rather high, due to the vacating of the $\mathrm{E}$ site. In other words, the first GTPconsuming step has made the A site highly restrictive. GTP hydrolysis ensues when a tc of EF-TuB ${ }_{0} \cdot$ GTP and either a cognate or a near-cognate aa-tRNA now interacts with the ribosome. Noncognate tc's, however, cannot enter the allosterically altered A site because they lack the conformation induced by codon-anticodon interaction. The relatively large number of noncognate tRNAs is thus excluded. This lack of the right conformation also holds them back from premature, noncoded elongation of the nascent chain in the initial stage when the E site is still occupied. 
What happens when a cognate or a near-cognate aa-tRNA enters the ribosome via step 1? Does a noncoded GTPase activation (Figure 1, step 2) also occur under these conditions? Studies by Swart et al. (1987) and by Talens et al.

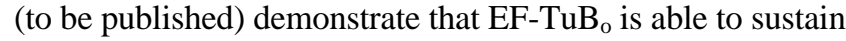
poly $(\mathrm{U})$ translation, but this requires elevated $\mathrm{Mg}^{2+}$ concentrations. EF-TuB $\mathrm{B}_{\mathrm{o}}$-mediated translation of natural mRNA is virtually absent under these conditions. Noncoded activation thus does not seem essential for a cognate tc to enter the elongation cycle at high $\mathrm{Mg}^{2+}$, but at the low $\mathrm{Mg}^{2+}$ concentrations optimizing natural RNA translation, it seems to be required. Rodnina and Wintermeyer (1995), working with wild-type EF-Tu, reported isolation of a quinary complex and hydrolysis of only one GTP molecule per peptide bond formed in two elongation rounds during translation of short heteromeric mRNAs with cognate tRNAs. On the other hand, studies over more than 20 elongation rounds of poly(U) translation by Weijland and Parmeggiani (1993, 1994) showed two GTP molecules being hydrolyzed by EF-Tu for each peptide bond formed. Although the question does not seem to be fully settled, perhaps entering of the ribosome by a cognate tc concomitant with clearance of the $\mathrm{E}$ site is permitted under certain conditions not fully known as yet.

The first GTP-consuming reaction may function as a "ribosomal screen" postulated by Gorini as early as 1971, although no underlying mechanism was offered. As recapitulated by Kurland (1979), such a screen would "allow binding of tRNA to the ribosome but antagonize tRNA from reaching the stage of codon-anticodon interaction". Active participation of the ribosome in tRNA selection as well as selection on the basis of tRNA conformation (Figure 1, steps 3 and 4) have been considered earlier by Kurland and coworkers $(1979,1990)$. This multistep selection process has to be completed by a final distinction between cognate and near-cognate tc's. Imperfect matching of codon and anticodon may result in a partial conformational change and less tight binding of near-cognate tc's to the restrictive A site during proofreading. Conformational selection may thus favor the binding of cognate over near-cognate tRNAs (vide infra).

In the classical scenario, all tc's compete through codonanticodon interactions. The free energy differences of these interactions are too small for discriminating between tRNAs. Hopfield (1974) and Ninio (1975) therefore proposed the mechanism of proofreading. It implies that the accuracy of the selection can be amplified through repetition of the selection event away from equilibrium, if it is driven by an extra free energy source, GTP hydrolysis. Experimental support for proofreading has been reported (Thompson, 1988; Ruusala et al., 1982b), but the relatively high accuracy in the initial selection step preceding GTP hydrolysis remained unexplained (Thompson \& Dix, 1982).

In the present model, which does not exclude proofreading, a discriminatory role is ascribed to conformational differences of cognate and near-cognate tRNAs induced through tRNAmRNA interaction. They may involve various elements of the overall tRNA structure. As the energy differences of codon-anticodon interaction are small, so are these structural differences. However, the conformational tRNA selection is driven by the extra free energy source of GTP hydrolysis, which in this case is used for creating a highly restrictive A site. The selective power of small conformational differences is thus amplified by the constraints of the A site. The high accuracy of the initial tRNA selection observed in vitro preceding GTP hydrolysis (Thompson \& Dix, 1982) may become explainable when tc binding to ribosomes with an empty $\mathrm{E}$ site and a highly restrictive A site was studied.

The scheme of the elongation cycle outlined so far implies that it is EF-TuB ${ }_{0}$ which causes the translational error. If so, why then is the combination of wild-type EF-Tu and EF$\mathrm{TuB}_{\mathrm{o}}$ uncapable of doing so? A plausible answer is that $\mathrm{EF}^{-\mathrm{TuB}_{\mathrm{o}}}$ is defective in its interaction with the ribosome (vide supra) and during this interaction is forced out by competition with its wild-type counterpart. EF-TuA $A_{R}$ binding to the ribosome is also relatively weak. It does not compete with $\mathrm{EF}-\mathrm{TuB}_{\mathrm{o}}$ to the same extent as wild-type EF-Tu. This makes EF-TuA $A_{R}$ the right complementing partner of EF-TuB in suppression, although it does not participate directly in the miscoding act.

A relevant question is whether the suppression data discussed here reflect basic steps of a normal elongation cycle. The translational errors may be due to a shorter residence on the ribosome of mutant tc's than that of the wild-type tc (van der Meide et al., 1980). If so, this is a kinetic effect but no essential deviation from the classical reaction scheme. $\mathrm{EF} \mathrm{TuB}_{\mathrm{o}}$ participates in normal elongation reactions up to a certain point, whereafter it fails to complete the elongation cycle. We cannot exclude that the concomitant action of two defective factors causes a deviation from the classical elongation pathway. Evidence for this, if any, is hard to interpret, however.

Mutant EF-Tu species have been instrumental in considering this revised, albeit perhaps incomplete, elongation scheme. In the wild-type cell, a functional distinction between two identical EF-Tu molecules operative in the cycle is problematic. The cooperation of two different defective factor molecules, one of which by itself is virtually unable to sustain protein synthesis, calls for a revision of the classical scheme of the cycle.

\section{ACKNOWLEDGMENT}

Our thanks are due to A. Parmeggiani, B. Kraal, A. P. Potapov, and R. Hilgenfeld for stimulating discussions and to E. L. H. Vorstenbosch for help with the preparation of the manuscript.

\section{REFERENCES}

Anborgh, P. H., \& Parmeggiani, A. (1991) EMBO J. 10, 779784.

Bensch, K., Pieper, U., Ott, G., Schirmer, N. K., Sprinzl, M., \& Pingoud, A. (1991) Biochimie 73, 1045-1050.

Duisterwinkel, F. J., de Graaf, J. M., Schretlen, P. J. M., Kraal, B., \& Bosch, L. (1981) Eur. J. Biochem. 117, 7-12.

Ehrenberg, M., Rojas, A.-M., Weiser, J., \& Kurland, C. G. (1990) J. Mol. Biol. 211, 739-749.

Ehrenberg, M., Bilgin, N., \& Scoble, J. (1993) in The Translational Apparatus: Structure, Function, Regulation, Evolution (Nierhaus, K. H., Franceschi, F., Subramanian, A. R., Erdmann, V. A., \& Wittmann-Liebold, B., Eds.) pp 305-315, Plenum Press, New York.

Gorini, L. (1971) Nature, New Biol. 234, 261-264.

Gorini, L. (1974) in Ribosomes (Nomura, M., Tissiere, A., \& Lengyel, P., Eds.) pp 791-803, Cold Spring Harbor Laboratory Press, Plainview, NY.

Hopfield, J. J. (1974) Proc. Natl. Acad. Sci. U.S.A. 71, 4135-4139. Hughes, D. (1987) J. Mol. Biol. 197, 611-615. 
Hughes, D., Atkins, J. F., \& Thompson, S. (1987) EMBO J. 6 4235-4239.

Kaziro, Y. (1990) Annu. Rev. Biochem. 60, 349-400.

Kurland, C. G. (1979) in Ribosomes, Structure, Function and Genetics (Chambliss, G., Craven, G. R., Davies, J., Davis, K., Kahan, L., \& Nomura, M., Eds.) pp 597-614, University Park Press, Baltimore.

Kurland, C. G., Jörgensen, F., Richter, A., Ehrenberg, M., Bilgin, N., \& Rojas, A. M. (1990) in The Ribosome: Structure, Function and Evolution (Hill, W. E., Dahlberg, A., Garrett, R. A., Moore, P. B., Schlesssinger, D., \& Warner, J. R., Eds.) pp 513-526, American Society for Microbiology, Washington, DC.

Miller, D. L., \& Weissbach, H. (1977) in Molecular Mechanisms of Protein Biosynthesis (Weissbach, H., \& Pestka, S., Eds.) pp 323-372, Academic Press, New York.

Nierhaus, K. H. (1993) Mol. Microbiol. 7, 661-669.

Ninio, J. (1975) Biochimie 57, 587-595.

Ozeki, H., Inokuchi, H., Yamao, F., Kodaira, M., Sakano, H., Ikemura, T., \& Shimura, Y. (1980) in Transfer RNA; Biological Aspects (Söll, D., Abelson, J. N., \& Schimmel, P. R., Eds.) p 341, Cold Spring Harbor Laboratory Press, Plainview, NY.

Rodnina, M. V., \& Wintermeyer, W. (1995) Proc. Natl. Acad. Sci. U.S.A. 92, 1945-1949.

Rodnina, M. V., Fricke, R., Kuhn, L., \& Wintermeyer, W. (1995) EMBO J. 14, 2613-2619.

Ruusala, T., Ehrenberg, M., \& Kurland, C. G. (1982a) EMBO J. 1, 75-78.

Ruusala, T., Ehrenberg, M., \& Kurland, C. G. (1982b) EMBO J. $1,741-745$.

Sam, T., Pingoud, A., \& Bosch, L. (1985) FEBS Lett. 185, 51-56.

Scoble, J., Bilgin, N., \& Ehrenberg, M. (1994) Biochimie 76, 5962.
Swart, G. W. M. (1987) Ph.D. Thesis, Leiden University, Leiden, The Netherlands.

Swart, G. W. M., Kraal, B., Bosch, L., \& Parmeggiani, A. (1982) FEBS Lett. 142, 101-106.

Swart, G. W. M., Parmeggiani, A., Kraal, B., \& Bosch, L. (1987) Biochemistry 26, 2047-2054.

Talens, A., Boon, K., Kraal, B., \& Bosch, L. (to be published).

Tapio, S., \& Kurland, C. G. (1986) Mol. Gen. Genet. 205, 186188.

Thompson, R. C. (1988) Trends Biochem. Sci. 13, 91-93.

Thompson, R. C., \& Dix, D. B. (1982) J. Biol. Chem. 257, 66776682.

Triana, F. J., Nierhaus, K. H., Ziehler, J., \& Chakraburtty (1993) in The Translational Apparatus: Structure, Function, Regulation, Evolution (Nierhaus, K. H., Fransceschi, F., Subramanian, A. R., Erdmann, V. A., \& Wittmann-Liebold, B., Eds.) pp 327338, Plenum Press, New York.

Van de Klundert, J. A. M., van der Meide, P. H., van de Putte, P., \& Bosch, L. (1978) Proc. Natl. Acad. Sci. U.S.A. 75, 44704473.

Van der Meide, P. H., Borman, T. H., van Kimmenade, A. M. A., van de Putte, P., \& Bosch, L. (1980) Proc. Natl. Acad. Sci. U.S.A. 77, 3922-3926.

Vijgenboom, E., \& Bosch, L. (1989) J. Biol. Chem. 264, 1301213017.

Vijgenboom, E., Vink, T., Kraal, B., \& Bosch, L. (1985) EMBO J. 4, 1049-1052.

Weijland, A., \& Parmeggiani, A. (1993) Science 259, 1311-1314.

Weijland, A., \& Parmeggiani, A. (1994) Trends Biochem. Sci. 19, $188-193$.

BI952925A 